\title{
Accuracy Improvement of Blood Glucose Measurement System Using Quantum Cascade Lasers
}

\author{
Takuya Koyama, Saiko Kino, Yuji Matsuura \\ Graduate School of Biomedical Engineering, Tohoku University, Sendai, Japan \\ Email: yuji@ecei.tohoku.ac.jp
}

How to cite this paper: Koyama, T., Kino, S. and Matsuura, Y. (2019) Accuracy Improvement of Blood Glucose Measurement System Using Quantum Cascade Lasers. Optics and Photonics Journal, 9, 155-164. https://doi.org/10.4236/opj.2019.910014

Received: September 3, 2019

Accepted: September 22, 2019

Published: September 25, 2019

Copyright (๑) 2019 by author(s) and Scientific Research Publishing Inc. This work is licensed under the Creative Commons Attribution International License (CC BY 4.0).

http://creativecommons.org/licenses/by/4.0/ (C) (i) Open Access

\begin{abstract}
For non-invasive measurement of blood glucose levels, a measurement system based on mid-infrared, attenuated-total-reflection spectroscopy equipped with hollow optical fibers, a trapezoidal multi-reflection prism, and two fixedwavelength quantum cascade lasers emitting different wavelengths is proposed. From the absorption spectra of lip mucosa measured by Fouriertransform infrared spectrometry, two wavelengths, $1152 \mathrm{~cm}^{-1}$ for absorption by glucose and $1186 \mathrm{~cm}^{-1}$ for the background, were chosen. To reduce measurement errors, the power distribution on the prism surface was investigated, and it was found that some high-intensity spots appear on the prism surface due to the coherency of the laser beam. This inhomogeneous power distribution causes measurement errors for slight movements of the lip mucosa. To homogenize the intensity distribution on the prism, a lens to excite higher modes in the fiber was introduced, and the incident angle was changed to suppress interference due to back-reflected light. These improvements increased the measurement stability, and in-vivo experiments demonstrated that the measured optical absorption correlates well with blood glucose levels.
\end{abstract}

\section{Keywords}

Blood Glucose, Infrared Spectroscopy, Quantum Cascade Laser, Hollow Optical Fiber

\section{Introduction}

Current blood glucose measurements require blood sampling by skin puncture, resulting in pain and risk of infection. Therefore, various non-invasive techniques, including infrared spectroscopy, for measuring blood glucose levels have 
been proposed [1] [2] [3] [4]. The most common methods developed thus far are based on near-infrared spectroscopy; however, these methods have limited accuracy because they detect the harmonic overtones and combination tones of molecular vibrations of glucose [5]. In contrast, methods based on mid-infrared light, a region in which glucose shows more isolated and strong absorption, enable more accurate measurements of blood glucose. Shen et al. applied mid-infrared spectroscopy for the measurement of human whole blood [6], and several groups have measured glucose in interstitial fluid by measuring the absorption spectra of fingertip [7] and oral mucosa [8] [9]. We previously proposed a measurement system based on infrared attenuated-total-reflection (ATR) spectroscopy, composed of a Fourier-transform infrared (FT-IR) spectrometer and flexible hollow optical fibers that transmit mid-infrared light. From the absorption spectra measured for human lips, we confirmed clear fingerprint spectra of glucose, and blood glucose levels were estimated with high accuracy by focusing on the glucose absorption peak originating from the pyranose structure of glucose [10] [11].

Although these reports have demonstrated the feasibility of mid-infrared spectroscopy for blood glucose measurements, systems involving an FT-IR spectrometer tend to be large and expensive; thus, a reduction in the system size and cost is necessary for the development of a simple health-care tool.

Recently, the development of quantum cascade lasers (QCLs), which enable the downsizing of mid-infrared spectroscopy systems, has advanced rapidly. Several groups have reported the application of QCLs to the measurement of blood glucose levels. Brandstetter et al. developed a system for measuring glucose, lactate, and triglyceride levels in blood serum based on an external cavity (EC)-QCL that is tunable in the glucose fingerprint region $\left(1030-1230 \mathrm{~cm}^{-1}\right)$ [12] [13]. The authors analyzed the absorption spectra of blood serum using partial-least-squares regression and determined blood glucose levels with a prediction error of $6.9 \mathrm{mg} / \mathrm{dL}$. Liakat et al. reported a good correlation between blood glucose levels and the spectra of backscattered light from human skin [14] [15]. These researchers irradiated the human palm or wrist with an EC-QCL and measured the backscattered light using a hollow optical fiber [14] or an integrating sphere [15]. Furthermore, some groups have used photoacoustic techniques to measure glucose concentrations in the interstitial fluid of human skin [16] [17] [18] [19] [20]. Recently, Pleitez et al. proposed the use of photothermal deflectometry enhanced by total internal reflection for blood glucose measurement [21]. In their system, visible laser light is used as a probe, and absorption on the surface of a Dove prism is detected to determine changes in the deflection angle of the probing light. This development can largely reduce the cost of mid-infrared detectors. Isensee et al. proposed an optofluidic measurement device based on a wavelength-tunable QCL for the continuous monitoring of glucose [22]. They used a miniaturized optofluidic interface in transflection geometry and performed in-vitro measurement of aqueous solutions containing glucose. 
As described above, numerous groups have developed blood glucose measurement systems based on QCLs; however, most of these systems use wavelength-tunable QCLs, which are expensive because a movable grating is required. The grating device also makes the system large and relatively unstable, thus requiring careful handling. Petrich's group proposed a system using a single Fabry-Perot QCL that emits a wide wavelength range covering the absorption band of glucose [23] [24]. The authors successfully measured glucose concentrations in the interstitial fluid of rats by using a fiber probe with small gaps that was filled with the sample material. Our group has proposed a system using common distributed-feedback (DFB)-QCLs giving a single narrow emission line. The use of DFB-QCLs can simplify the system, and a preliminary study demonstrated the possibility of blood glucose measurement using the proposed system [25] [26] [27]. In this paper, we reduce the measurement error of the QCL-based system and report the results of in-vivo measurements of blood glucose levels based on mid-infrared ATR spectroscopy.

\section{In-Vivo Measurement of Blood Glucose Levels}

Figure 1 shows the experimental setup for ATR measurement with two QCLs [27]. The output power of the DFB-QCLs (Sumitomo Electric) is approximately $5-10 \mathrm{~mW}$ in the continuous mode. An aspheric lens with a focal length of 5.95 $\mathrm{mm}$ is used to collimate the laser beam, and a hollow optical fiber (inner diameter: $2 \mathrm{~mm}$; length: $26 \mathrm{~cm}$ ) delivers the beam to the ATR prism. Based on our previous investigation, we chose two QCLs with wavenumbers of 1152 and 1186 $\mathrm{cm}^{-1}$ [27]. The wavenumber of $1152 \mathrm{~cm}^{-1}$ corresponds to an absorption peak of glucose solution that originates from the pyranose ring structure [28]. On the other hand, at around the wavenumber of $1186 \mathrm{~cm}^{-1}$, the absorption of glucose solution becomes minimal. Therefore, we have shown that the differential absorption between 1152 and $1186 \mathrm{~cm}^{-1}$ calculated from the lip's absorption spectra measured by using a FT-IR based system showed a good correlation with blood glucose levels measured by blood sampling.

We utilized a trapezoidal ATR prism made of zinc sulfide, which is nontoxic and highly transparent in the mid-infrared region. The thickness of the prism is $1.6 \mathrm{~mm}$, and the length is set to $24 \mathrm{~mm}$ to be suitable for human lips. The prism

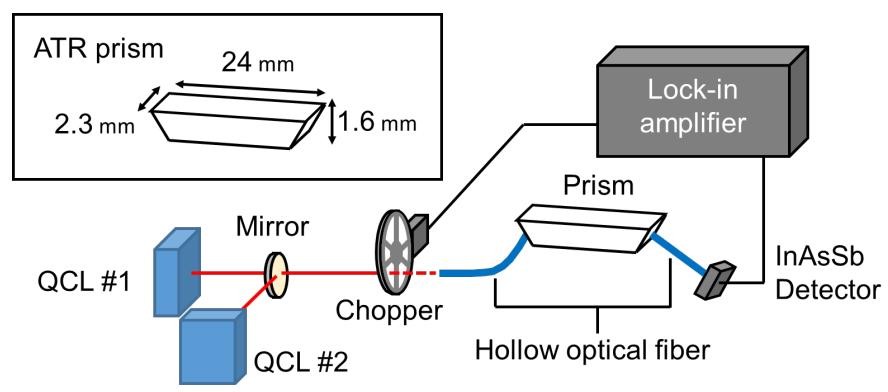

Figure 1. Schematic of the experimental setup based on two QCLs for measuring the optical absorption of lip mucosa. 
gives 15 reflections at the top and bottom surfaces, and the output beam from the prism is detected by an InAsSb semiconductor detector. In our in-vivo measurement, the two QCLs were switched using a flipper mirror. For each wavelength, the detected power was averaged for $5 \mathrm{~s}$. In addition to the optical measurement, the blood glucose levels were measured by blood sampling using a self-monitoring-of-blood-glucose system (J\&J, One Touch VerioVue). Our protocol was approved by the ethical committee on the Use of Humans as Experimental Subjects of Tohoku University (No. 15A-3), and informed consent was obtained from the examinee.

We firstly compared the measurement errors of the QCL system with those of the FT-IR system. Figure 2 shows the differential absorption between 1152 and $1186 \mathrm{~cm}^{-1}$ from 25 consecutive measurements using the QCL and FT-IR systems. These absorptions are normalized to have an average of zero and clearly illustrate that the measurement errors are much larger in the QCL system. Therefore, we investigated the cause of the error in the QCL system to suppress the measurement error.

One of the greatest differences in the optical beams used in the QCL and FT-IR systems is the coherency. The incoherent beam used in the FT-IR has a large beam divergence, which results in more uniform irradiation of the prism surface. In contrast, the coherent beam of the QCL transmits a single ray onto the prism, which may cause the light intensity to be locally concentrated on the surface of the prism as hot spots. Furthermore, a portion of the incident light is reflected by the end of the prism, and the forward and backward beams interfere with each other, thus making the localized spots more distinct.

For this reason, we performed an experiment to confirm the existence of hot spots in the QCL system. We partially masked the upper surface of the prism with polytetrafluoroethylene tape that absorbs mid-infrared light and measured the optical loss due to the absorption of the tape strip. Figure 3 shows the optical loss measured when different parts of the upper surface are masked by tape with a width of $2 \mathrm{~mm}$. The estimated reflection points of the light are also shown in Figure 3, and we found that the losses are high when the reflection points are

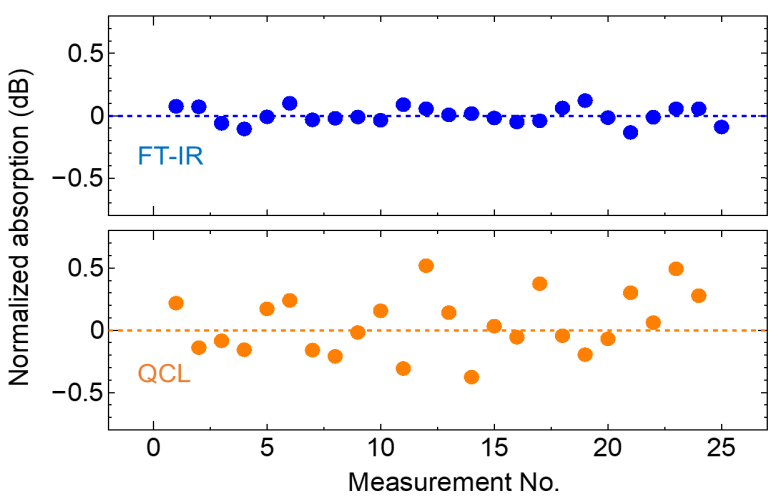

Figure 2. Measurement errors in the differential absorption between 1152 and $1186 \mathrm{~cm}^{-1}$ for the FT-IR and QCL systems. 


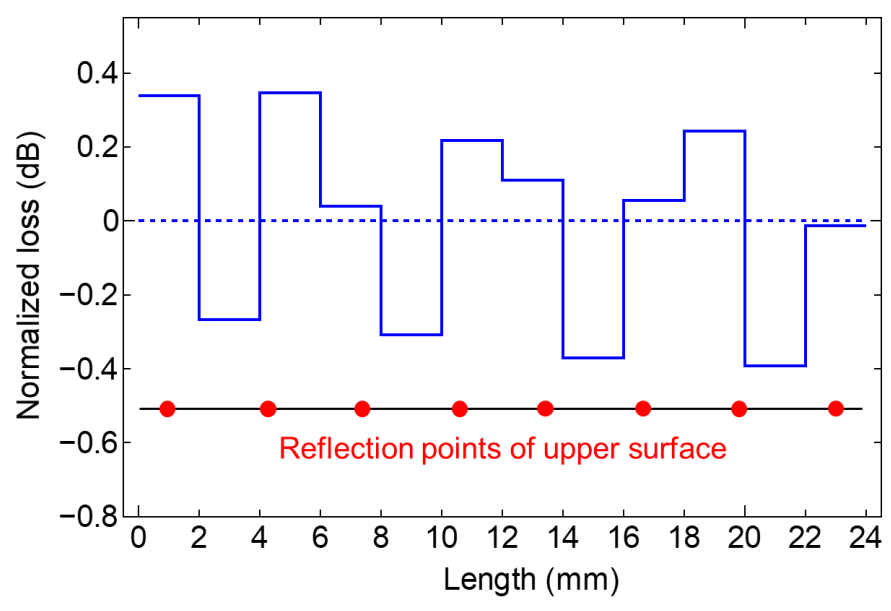

Figure 3. Measured loss when the upper surface of the prism is partially masked by an absorber.

masked. This result demonstrates that the light is concentrated at the reflection points, leading to hot spots. This behavior can cause large errors because the measured absorption changes when the lip shifts slightly. Because mucosal tissue is not highly uniform, more uniform irradiation is necessary.

To resolve this matter, we attempted to homogenize the intensity distribution of the light on the prism surface. First, we introduced a lens with a short focal length $(\mathrm{f}=5.95 \mathrm{~mm})$ prior to the lead-in fiber. Because a number of higher-order modes are excited in the fiber, the divergence angle of the incident beam on the prism becomes large. In addition, we increased the angle of the incident light on the prism by tilting the fiber 10 degrees with respect to the end of the prism to prevent multi-reflection interference. Figure 4 shows the losses measured when the upper surface of the prism is partially masked by an absorber after applying the above improvements. One can see that the light intensity on the prism surface is homogenized and that the hot spots observed in Figure 3 have almost completely disappeared.

To confirm the effect of the homogenization, we measured the lip absorption using the improved system. The differential absorptions of 25 consecutive measurements measured by the original and improved systems are shown in Figure 5. We confirmed that the measurement errors are clearly suppressed due to the improvement of the system. The standard deviations before and after the improvement are $0.33 \mathrm{~dB}$ and $0.087 \mathrm{~dB}$, respectively; and from this result, we confirmed the efficacy of the improvement.

Finally, we performed in-vivo measurements using the improved system. Figure 6 shows the differential absorption between 1152 and $1186 \mathrm{~cm}^{-1}$ and the blood glucose level. The changes in the optical absorption closely follow the changes in the blood glucose level. Figure 7 shows results for nine series of measurements performed for a single subject. For each series, the optical absorption of the lips and the blood glucose level were measured for $120 \mathrm{~min}$ after a meal, with an interval of approximately $10 \mathrm{~min}$. The results for six series of 


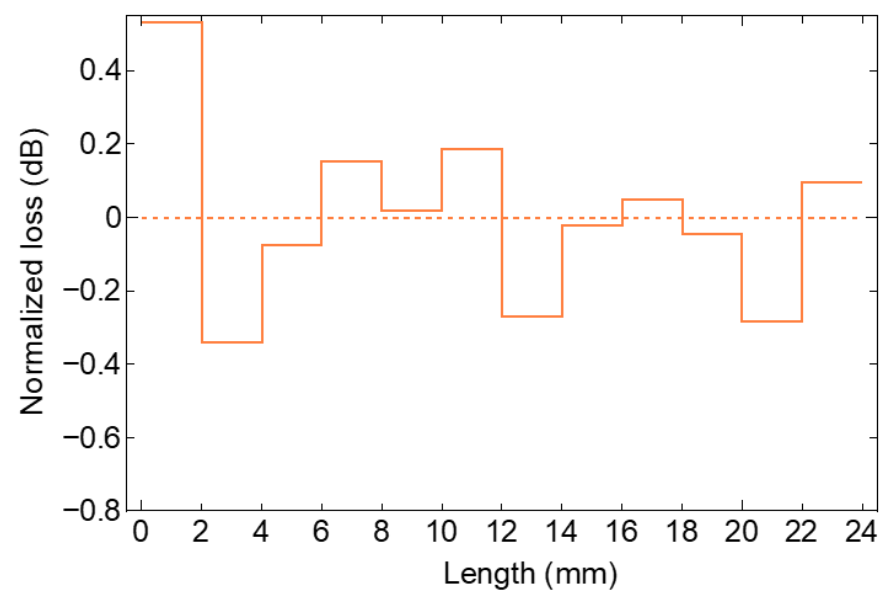

Figure 4. Losses measured when the upper surface of the prism is partially masked by an absorber after applying improvements.

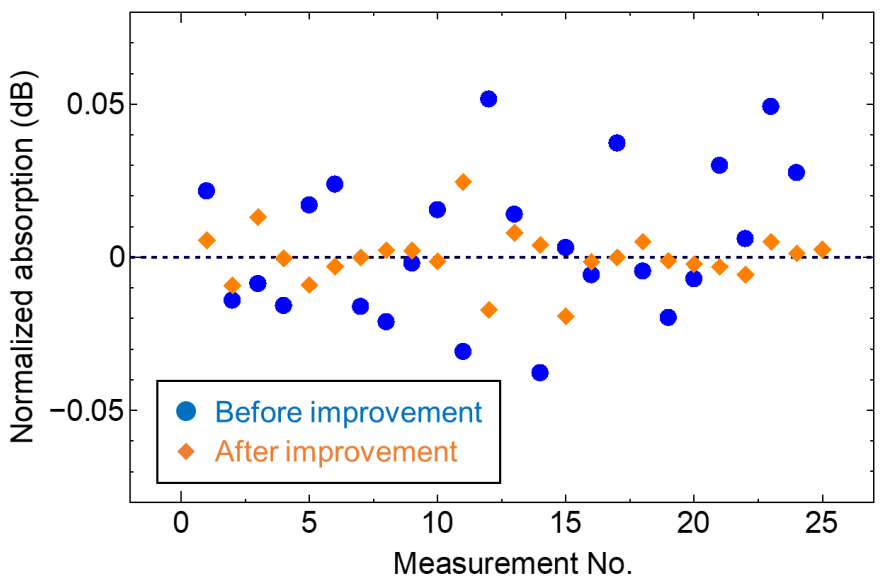

Figure 5. Differential absorption between 1152 and $1186 \mathrm{~cm}^{-1}$ for 25 consecutive lip measurements using the original and improved systems.

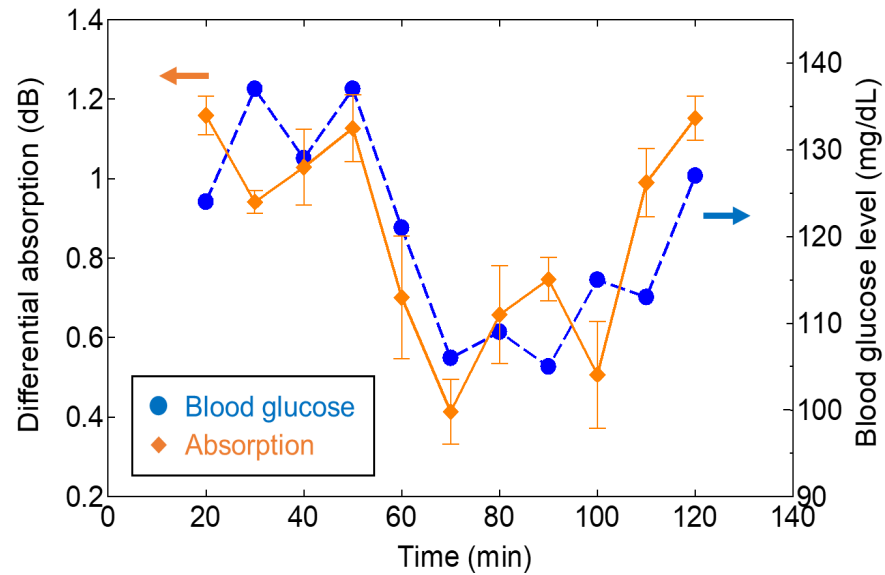

Figure 6. Changes in the differential absorption between 1152 and $1186 \mathrm{~cm}^{-1}$ measured by the improved system. Changes in the bloodglucose level are also shown for comparison. 


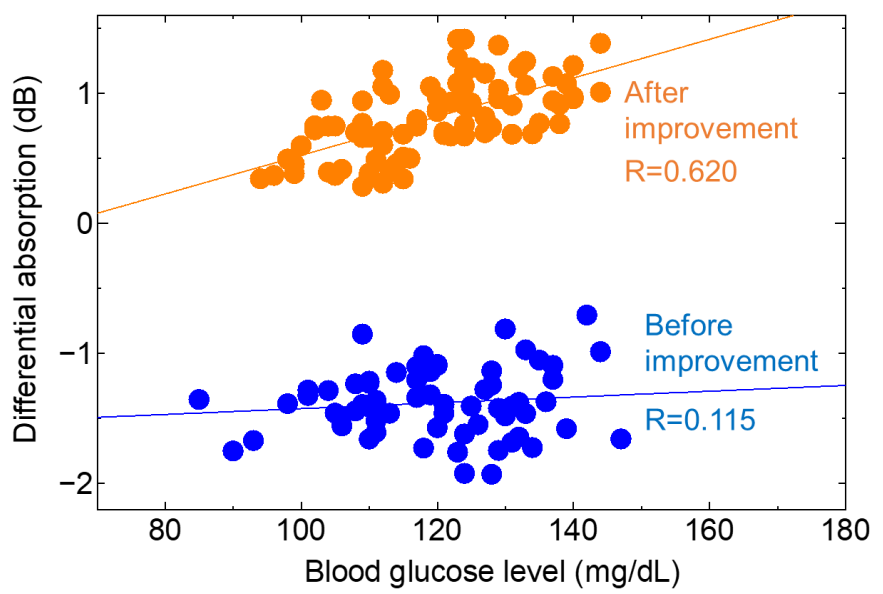

Figure 7. Correlation between the absorption and blood glucose level measured using the original and improved systems.

measurements performed using the system before the improvement are also shown, and a better correlation, with a coefficient of $\mathrm{R}=0.620$, is observed for the improved system. Moreover, the sensitivity is improved because the regression coefficient was increased by the improvement. This result occurred because the incident angle of the laser light on the prism was changed, which reduced the incident angle on the prism surface and increased the penetration depth of the evanescent light. We found that the optically estimated values of the improved system are within $20 \%$ of the reference values. Therefore, we confirmed the feasibility of non-invasive glucose measurement with two fixed-wavelength QCLs.

\section{Conclusions}

We developed a blood glucose measurement system based on mid-infrared ATR spectroscopy with two fixed-wavelength QCLs. Based on the absorption spectra of lip mucosa measured by FT-IR, two wavelengths, $1152 \mathrm{~cm}^{-1}$ for glucose absorption and $1186 \mathrm{~cm}^{-1}$ for the background, were chosen. We performed in-vivo measurements using the ATR system equipped with two single-wavelength QCLs and we found that the measurement errors strongly affected the measurement accuracy. To suppress the measurement error, the power distribution on the prism surface is investigated. When we partially masked the upper surface of the prism with an optical absorber, the surface points exhibiting high optical loss coincided with the geometric reflection points. Thus, we introduced a lens that excites higher modes of the light in the prism to diffuse the light and tilted the fiber by 10 degrees with respect to the end of the prism to prevent multiple reflections. We confirmed that the intensity distribution on the prism was homogenized and that the absorption errors for the lips were suppressed in the improved system.

In the in-vivo measurement results for the improved system, the change in absorption follows the change in blood glucose level better than before the improvement. In measurements acquired over nine days, we found a good correla- 
tion, with a coefficient of 0.62 , between the absorption and the blood glucose level, confirming the feasibility of the proposed QCL system.

We are currently working on experiments with more subjects and further improvements to the shape of the prism. In addition, we are also working on optimizing the selected wavelength by using regression analysis or a neural network to improve accuracy [29] [30].

\section{Funding}

This research was funded by JSPS KAKENHI Grant Numbers JP17H03242 and JP18K18842.

\section{Acknowledgements}

The authors thank Sumitomo Electric Industries, Ltd. for providing QCLs used in the experiments.

\section{Conflicts of Interest}

The authors declare no conflicts of interest regarding the publication of this paper.

\section{References}

[1] Vashist, S.K. (2012) Non-Invasive Glucose Monitoring Technology in Diabetes Management: A Review. Analytica Chimica Acta, 750, 16-27. https://doi.org/10.1016/j.aca.2012.03.043

[2] Oliver, N.S., Toumazou, C., Cass, A.E. and Johnston, D.G. (2009) Glucose Sensors: A Review of Current and Emerging Technology. Diabetic Medicine, 26, 197-210. https://doi.org/10.1111/j.1464-5491.2008.02642.x

[3] So, C., Choi, K., Wong, T.K., and Chung, J.W. (2012) Recent Advances in Noninvasive Glucose Monitoring. Medical Devices, 5, 45-52. https://doi.org/10.2147/MDER.S28134

[4] Kondepati, V.R. and Heise, H.M. (2007) Recent Progress in Analytical Instrumentation for Glycemic Control in Diabetic and Critically Ill Patients. Analytical and Bioanalytical Chemistry, 388 545-563. https://doi.org/10.1007/s00216-007-1229-8

[5] Yadav, J., Rani, A., Singh, V. and Murari, B.M. (2015) Prospects and Limitations of Non-Invasive Blood Glucose Monitoring Using Near-Infrared Spectroscopy. Biomedical Signal Processing and Control, 18, 214-217. https://doi.org/10.1016/j.bspc.2015.01.005

[6] Shen, Y.C., Davies, A.G., Linfield, E.H., Elsey, T.S., Taday, P.F. and Arnone, D.D. (2003) The Use of Fourier-Transform Infrared Spectroscopy for the Quantitative Determination of Glucose Concentration in Whole Blood. Physics in Medicine and Biology, 48, 2023-2032. https://doi.org/10.1088/0031-9155/48/13/313

[7] Chen, J.Y., Zhou, Q., Xu, G., Wang, R.T., Tai, E.G., Xie, L., et al. (2019) Non-Invasive Blood Glucose Measurement of 95\% Certainty by Pressure Regulated Mid-IR. Talanta, 197, 211-217. https://doi.org/10.1016/j.talanta.2019.01.034

[8] Heise, H.M. and Marbach, R. (1998) Human Oral Mucosa Studies with Varying Blood Glucose Concentration by Non-Invasive ATR-FT-IR-Spectroscopy. Cellular and Molecular Biology, 44, 899-912. 
[9] Kajiwara, K., Uemura, T., Kishikawa, H., Nishida, K., Hashiguchi, Y., Uehara. M., et al. (1993) Noninvasive Measurement of Blood Glucose Concentrations by Analyzing Fourier Transform Infra-Red Absorbance Spectra Through Oral Mucosa. Medical \& Biological Engineering \& Computing, 31, S17-S22. https://doi.org/10.1007/BF02446645

[10] Kino, S., Tanaka, Y. and Matsuura, Y. (2014) Blood Glucose Measurement by using Hollow Optical Fiber-Based Attenuated Total Reflection Probe. Journal of Biomedical Optics, 19, Article No. 057010. https://doi.org/10.1117/1.JBO.19.5.057010

[11] Kino, S., Omori, S., Katagiri, T. and Matsuura, Y. (2016) Hollow Optical-Fiber Based Infrared Spectroscopy for Measurement of Blood Glucose Level by Using Multi-Reflection Prism. Biomedical Optics Express, 7, 701-708.

https://doi.org/10.1364/BOE.7.000701

[12] Brandstetter, M., Genner, A., Anic, K. and Lendl, B. (2010) Tunable External Cavity Quantum Cascade Laser for the Simultaneous Determination of Glucose and Lactate in Aqueous Phase. Analyst, 135, 3260-3265.

https://doi.org/10.1039/c0an00532k

[13] Brandstetter, M., Volgger, L., Genner, A., Jungbauer, C. and Lendl, B. (2013) Direct Determination of Glucose, Lactate and Triglycerides in Blood Serum by a Tunable Cascade Laser-Based Mid-IR Sensor. Applied Physics B, 110, 233-239. https://doi.org/10.1007/s00340-012-5080-Z

[14] Liakat, S., Bors, K.A., Xu, L., Woods, C.M., Doyle, J. and Gmachl, C.F. (2014) Noninvasive in Vivo Glucose Sensing on Human Subjects Using Mid-Infrared Light. Biomedical Optics Express, 5, 2397-2404. https://doi.org/10.1364/BOE.5.002397

[15] Werth, A., Liakat, S., Dong, A., Woods, C.M. and Gmachl, C.F. (2018) Implementation of an Integrating Sphere for the Enhancement of Noninvasive Glucose Detection Using Quantum Cascade Laser Spectroscopy. Applied Physics B, 124, 75. https://doi.org/10.1007/s00340-018-6946-5

[16] Pleitez, M., Von Lilienfeld-Toal, H. and Mäntele, W. (2013) Infrared Spectroscopic Analysis of Human Interstitial Fluid in Vitro and in Vivo Using FT-IR Spectroscopy and Pulsed Quantum Cascade Lasers (QCL): Establishing a New Approach to Non Invasive Glucose Measurement. Spectrochimica Acta Part A, 85, 61-65. https://doi.org/10.1016/j.saa.2011.09.007

[17] Von Lilienfeld-Toal, H., Weidenmüller, M., Xhelaj, A. and Mäntele, W. (2005) A Novel Approach to Non-Invasive Glucose Measurement by Mid-Infrared Spectroscopy: The Combination of Quantum Cascade Lasers (QCL) and Photoacoustic Detection. Vibrational Spectroscopy, 38, 209-215. https://doi.org/10.1016/j.vibspec.2005.02.025

[18] Pleitez, M.A., Lieblein, T., Bauer, A., Hertzberg, O., Von Lilienfeld-Toal, H. and Mäntele, W. (2013) In Vivo Noninvasive Monitoring of Glucose Concentration in Human Epidermis by Mid-Infrared Pulsed Photoacoustic Spectroscopy. Analytical Chemistry, 85, 1013-1020. https://doi.org/10.1021/ac302841f

[19] Kottmann, J., Rey, J.M., Luginbühl, J., Reichmann, E. and Sigrist, M.W. (2012) Glucose Sensing in Human Epidermis Using Mid-Infrared Photoacoustic Detection. Biomedical Optics Express, 3, 667-680. https://doi.org/10.1364/BOE.3.000667

[20] Kottmann, J., Rey, J.M. and Sigrist, M.W. (2016) Mid-Infrared Photoacoustic Detection of Glucose in Human Skin: Towards Non-Invasive Diagnostics. Sensors, 16, 1663. https://doi.org/10.3390/s16101663

[21] Pleitez, M.A., Hertzberg, O., Bauer, A., Seeger, M., Lieblein, T., Von Lilienfeld-Toal, H. and Mäntele, W. (2015) Photothermal Deflectometry Enhanced by Total Internal 
Reflection Enables Non-Invasive Glucose Monitoring in Human Epidermis. Analyst, 140, 483-488. https://doi.org/10.1039/C4AN01185F

[22] Isensee, K., Müller, N., Pucci, A. and Prtrich, W. (2018) Towards a Quantum Cascade Laser-Based Implant for the Continuous Monitoring of Glucose. Analyst, 143, 6025. https://doi.org/10.1039/C8AN01382A

[23] Vrančić, C., Fomichova, A., Gretz, N., Herrmann, C., Neudecker, S., Pucci, A. and Petrich, W. (2011) Continuous Glucose Monitoring by Means of Mid-infrared Transmission Laser Spectroscopy in Vitro. Analyst, 136, 1192-1198. https://doi.org/10.1039/c0an00537a

[24] Vrančić, C., Kruger, N.,Gretz, N., Neudecker, S., Pucci, A. and Petrich, W. (2014) A Quantitative Look Inside The Body: Minimally Invasive Infrared Analysis in Vivo. Analytical Chemistry, 86, 10511-10514. https://doi.org/10.1021/ac5028808

[25] Yoshioka, K., Kino, S. and Matsuura, Y. (2017) Noninvasive Measurement of Blood Glucose Level Using Mid-Infrared Quantum. Proceedings of the SPIE, 10251, $102511 \mathrm{U}$. https://doi.org/10.1117/12.2275006

[26] Yoshioka, K., Kino, S. and Matsuura, Y. (2018) Blood Glucose Measurement with Multiple Quantum Cascade Lasers Using Hollow-Optical Fiber-Based ATR Spectroscopy. Proceedings of the SPIE, 10488, 104880D. https://doi.org/10.1117/12.2288131

[27] Koyama, T., Kino, S. and Matsuura, Y. (2019) Non-Invasive Blood Glucose Measurement Using Fixed-Wavelength Quantum Cascade Lasers. Proceedings of the SPIE, 10872, 108720A. https://doi.org/10.1117/12.2508200

[28] Ibrahim, M., Alaam, M., El-Haes, H., Jalbout, A.F. and De Leon, A. (2006) Analysis of the Structure and Vibrational Spectra of Glucose and Fructose. Eclética Química, 31, 15-21. https://doi.org/10.1590/S0100-46702006000300002

[29] Kasahara, R., Kino, S., Soyama, S. and Matsuura, Y. (2017) Noninvasive Glucose Monitoring Using Mid-Infrared Absorption Spectroscopy Based on a Few Wavenumbers. Biomedical Optics Express, 9, 289-232.

https://doi.org/10.1364/BOE.9.000289

[30] Kasahara, R., Kino, S., Soyama, S. and Matsuura, Y. (2018) Unsupervised Calibration for Noninvasive Glucose-Monitoring Devices Using Mid-Infrared Spectroscopy. Journal of Innovative Optical Health Sciences, 11, Article No. 1850038. https://doi.org/10.1142/S1793545818500384 